Supporting Information

\title{
Raman Tweezers Microspectroscopy of Functionalized 4.2-nm Diameter CdSe Nanocrystals in Water Reveals Changed Ligand Vibrational Modes by a Metal Cation
}

Randa Mrad, ${ }^{1}$ Sergei G. Kruglik, ${ }^{2}$ Nassim Ben Brahim, ${ }^{1}$ Rafik Ben Chaâbane, ${ }^{1}$ and Michel Negrerie, ${ }^{*}, 3$

${ }^{1}$ Laboratoire des Interfaces et Matériaux Avancés, Faculté des Sciences de Monastir, Bd. de l'Environnement, 5019 Monastir, Tunisia

${ }^{2}$ Laboratoire Jean Perrin, Sorbonne Université, CNRS, 75005 Paris, France

${ }^{3}$ Laboratoire d'Optique et Biosciences, INSERM, CNRS, Ecole Polytechnique, 91128

Palaiseau, France

*E-mail: michel.negrerie@polytechnique.fr<smiles>N[C@@H](CS)C(O)O</smiles>

L-Cysteine<smiles>OCC(O)CS</smiles>

Thioglycerol

Figure S1. Structure of the two ligands used for capping the nanoparticles. 


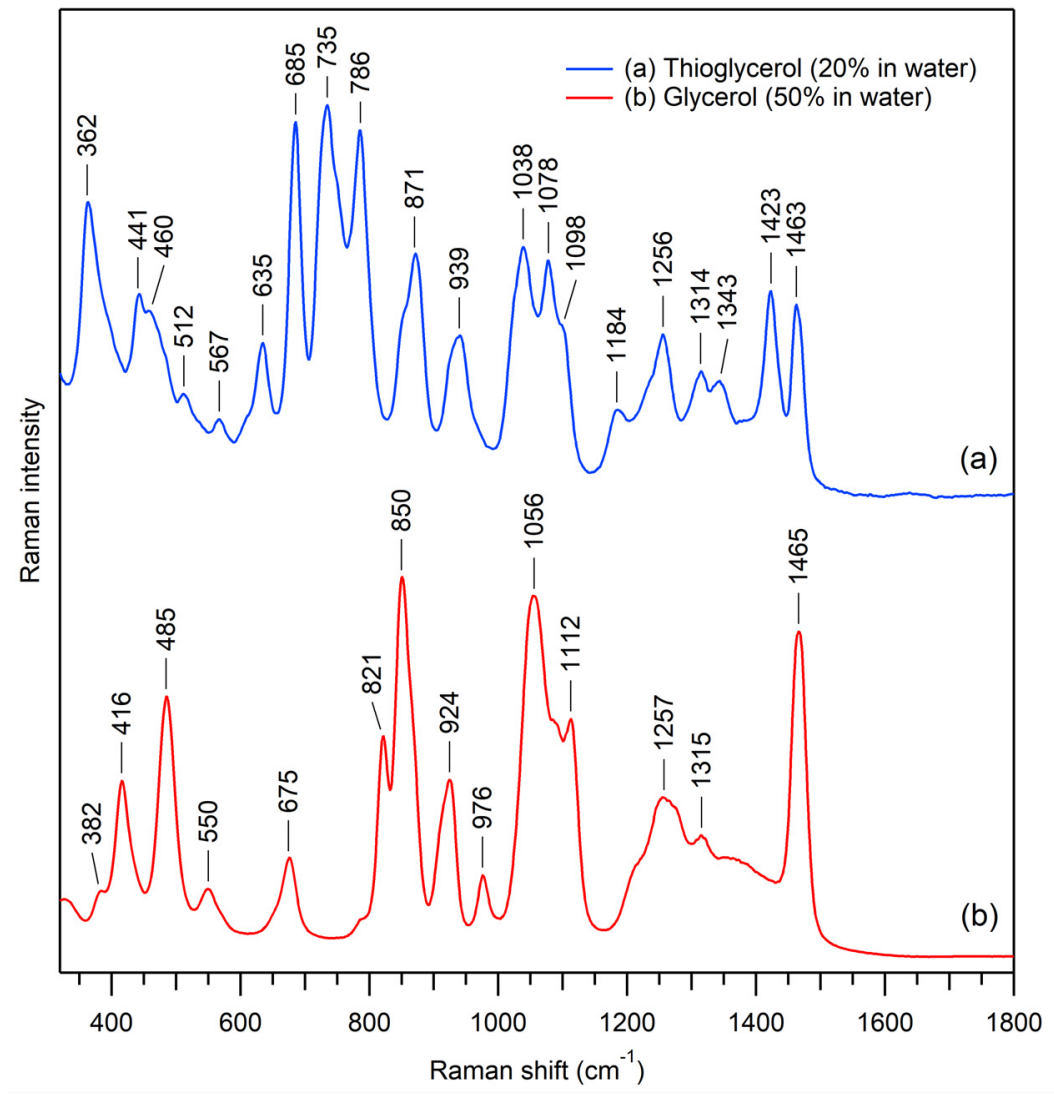

Figure S2. Comparison between the Raman spectra of glycerol at $50 \%$ in water and thioglycerol (3-mercapto-1,2-propanediol) at $20 \%$ in water.

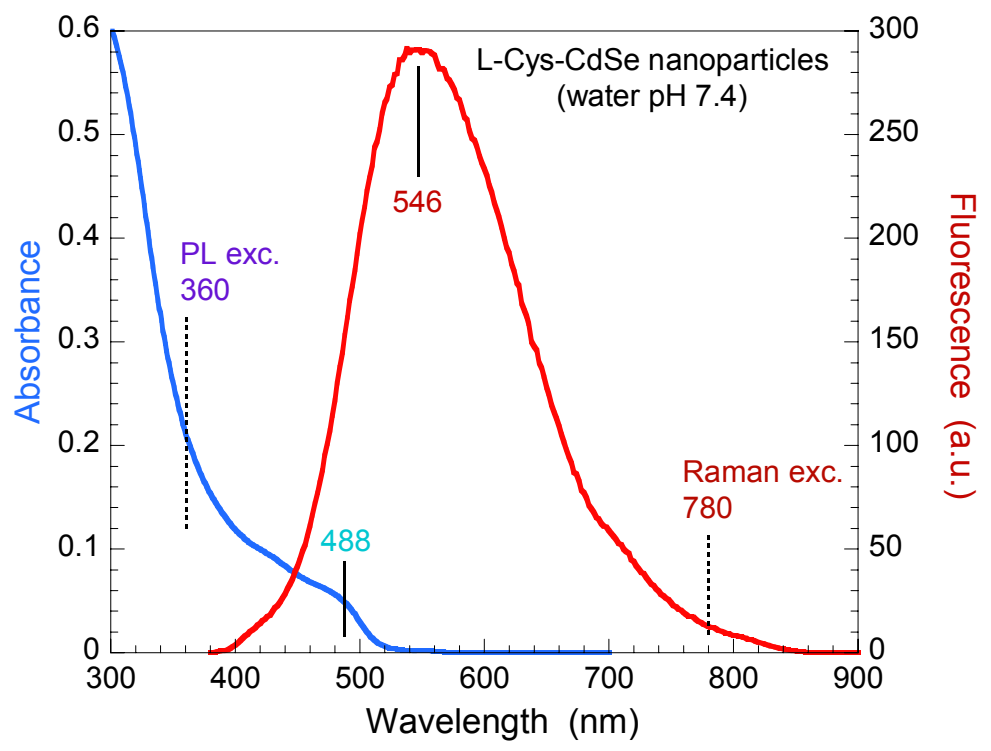

Figure S3. Absorption and fluorescence spectra of L-cysteine QDs in water. 